\title{
Limitations Regarding Use of the ABC-Model for Interpretation of Partial Discharge Measurements
}

\author{
T. G. Aakre, R. Skattenborg \& E. Ildstad \\ NTNU/ Department of Electrical Power engineering, Trondheim, Norway
}

\begin{abstract}
The main purpose of the work presented here is to facilitate use of partial discharge (PD) measurements as a diagnostic tool for condition assessment of hydropower generator bars. The main aim of the work is to clarify how measured inception and extinction voltage, detected number and magnitude of PDs relate to the void size and the physical discharge mechanisms in the voids. The laboratory tests were performed on $3 \mathrm{~mm}$ thick samples, consisting of three $1 \mathrm{~mm}$ thick layers containing a cylindrical disc shaped void in the center of the middle layer. The void diameters varied from 3 to $20 \mathrm{~mm}$. Insulation discs of polycarbonate and generator insulation based on mica and glass fiber reinforced epoxy tape were examined. In case of the polycarbonate samples comparable tests were performed on objects with either conductive or insulting void surfaces. During application of a stepwise increasing/decreasing $50 \mathrm{~Hz} \mathrm{AC}$ voltage, the partial discharge activity was detected using a conventional PRPDA detection system.
\end{abstract}

The inception voltage was found to decrease with increasing void diameter, in accordance with results from electric field calculations showing increased field enhancement in the larger voids. In all cases the apparent discharge magnitude increased with increasing diameter. In case of conducting void surfaces this discharge magnitude and number of PDs per period were, in accordance with the theory, found to be proportional to the total surface area of the void. In case of voids with insulating surfaces, this proportionality was valid up to a void diameter of $10 \mathrm{~mm}$. Larger voids resulted in higher number of PD each at lower apparent charge magnitude than expected, indicating several parallel discharges, during which a section of the void surface is discharged only. In contradiction to the assumptions of the abc-model nearly no difference was observed between the PD inception and extinction voltage, indicating a high and rapidly decaying remanent charge.

Thus, the abc-model assumption of each PD causing a complete void discharging is only fulfilled if the surfaces are conducting, or the voids are small. The results indicate that changes of internal conditions of the void during the period of PD measurements need to be considered in a more realistic model.

\section{Introduction}

Measurements of PD activity is one of the main tools used to assess the condition of generator insulation [1-3]. Such PD measurements are generally used to identify and distinguish different type of defects, for instance by analyzing the distributions of PD magnitudes and phase-resolved partial discharge pattern (PRPDA) [1]. Its suitability for diagnostic testing of large hydropower generator insulation is, however, questionable. In case of diagnostic testing of PD on generator bars, it is particularly challenging that the mixed insulation system itself, consisting of glass fiber and mica reinforced epoxy is characterized by high capacitance values and high PD activity, even in case of unaged insulation. Such an insulation system is, however, selected due to its excellent thermal and mechanical properties as well as its ability to endure high PD activity during service. It is therefore of crucial importance to identifying characteristic features and any changes of detectable PD activity, which can be used as a diagnostic tool for condition assessment of hydro generators.

The main purpose of the work presented here was to experimentally test the validity of assumptions commonly used to relate measurable PD parameters to selected dimensions and proposed discharge activity of the voids.

\section{The ABC model for interpretation of Partial Discharge measurements}

It is common to describe the PD activity of in a test object by the so-called abc-model [4]. In this model the void is considered a capacitance $C_{\mathrm{c}}$, whereas $C_{\mathrm{b}}$ is the capacitance of the insulation in series with the void, and $C_{\mathrm{a}}$ is the capacitance of the remaining insulation in parallel (see details of Figure 4). This latter capacitance includes both the sample and external stray capacitance.

The classical interpretation of measurable quantities using this abc-model is based upon the following assumptions:

1. The electric field in the void is homogeneous, varying only with dimensions, permittivity and applied voltage.

2. The complete void surface area is discharged in each PD event

3. Remanent charges from each PD event do not decay between each PD.

4. The internal conditions in the void, i.e. gas pressure and surface conductivity, remain unchanged during the period of PD measurement.

When a voltage $U_{\mathrm{a}}$ is applied, the capacitive voltage across the void, $U_{\text {void }}$, is given by:

$$
U_{\text {void }}=\frac{C_{\mathrm{b}}}{C_{\mathrm{b}}+C_{\mathrm{c}}} U_{\mathrm{a}}
$$

At a certain applied voltage, a PD occurs in the void. At this partial discharge inception voltage (PDIV) the voltage across the void is larger than the $\mathrm{AC}$ breakdown strength of the gas in the void. During such a discharge the voltage across the void abruptly changes from the ignition voltage $U_{\mathrm{s}}$ to the remanent voltage $U_{\mathrm{r}}$. In case of small voids, with homogeneous electric field the inception voltage is usually assumed to vary with the 
gap distance $d$ and gas pressure $p$ according to the Paschen law. In case of relatively large voids, valid for $p d$ values to the right of the minimum (in the range $10^{-2}$ to $5 \cdot 10^{2}$ [bar $\left.\mathrm{cm}\right]$ ), the following approximation is used [4]:

$$
U_{\mathrm{s}}=6.72 \sqrt{p d}+24.36(p d)[k V]
$$

The remanent voltage $U_{\mathrm{r}}$ is the voltage across the void at which the discharge extinguishes. The value of this voltage depends upon many local factors making it difficult to predict. In theory it can be determined by considering the number of complete discharges per period, using the following expression:

$$
n=\frac{2 \cdot\left(\frac{C_{\mathrm{b}}}{C_{\mathrm{b}}+C_{\mathrm{c}}} U_{\text {applied }}-U_{\mathrm{r}}\right)}{U_{\mathrm{s}}-U_{\mathrm{r}}}
$$

where the repetition rate $n$ is rounded down for non-integer numbers. The repetition rate is however expected to be linear with voltage rather than stepwise, as was shown in [5].

After a discharge has once started, the applied voltage may be reduced without the discharges disappearing. When the applied voltage is reduced to the PD extinction voltage (PDEV) the discharges ease. Just above this voltage one partial discharge is expected each time the voltage is at its peak value. Then a relation between extinction voltage and the remanent voltage is found by putting $n=1$ in equation (3).

In addition to these characteristics the apparent charge $q_{\mathrm{a}}$ is a most important measurable quantity. It is measured in the external circuit as the calibrated charge needed to restore the voltage across the test object immediately after a PD event:

$$
q_{\mathrm{a}}=\Delta U_{\mathrm{c}}\left(C_{\mathrm{a}}+\frac{C_{\mathrm{c}} C_{\mathrm{b}}}{C_{\mathrm{b}}+C_{\mathrm{c}}}\right)\left(\frac{C_{\mathrm{b}}}{C_{\mathrm{b}}+C_{\mathrm{a}}}\right)
$$

In case of small void capacitances compared to the total capacitance, the following approximation is considered valid:

$$
q_{\mathrm{a}} \approx \Delta U_{\mathrm{c}} \cdot C_{\mathrm{b}}
$$

The statistical time lag and the memory effect of previous discharges affect the discharge characteristics substantially; the PD magnitude, the number of discharges per voltage period and the phase angle at which the discharges occur are all affected. This manifest itself in the phase-resolved partial discharge pattern, which is being extensively used to interpret discharges for diagnostic of insulation systems [6].

The dissipated energy during a discharge can be used as a measure of the potential damage caused by a partial discharge. The dissipated energy from one discharge is found by the following formula:

$$
\Delta W=2 U_{e} \cdot q_{a}
$$

where $U_{e}$ is the extinction voltage. This justifies the use of apparent charge and extinction voltage as characteristic discharge parameters.

Two examples of void voltage are given in Figure 1 where a) is at PDIV and b) is at twice the PDIV with a correspondingly higher repetition rate. The PD magnitude remains unchanged in these cases. The green curve is the applied voltage, the purple curve the residual voltage and orange the corresponding void voltage as the summation of the green and purple curve.

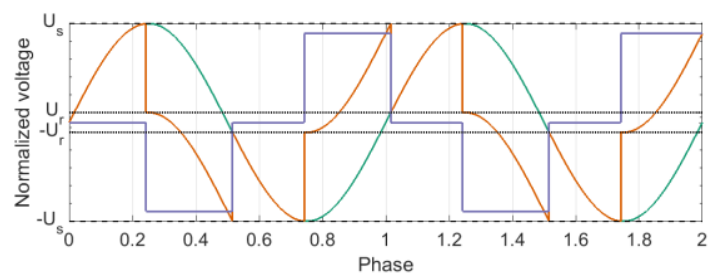

a) Applied voltage at PDIV for the first two periods. $n=2$ per half cycle in the second period when remanent charges are present.

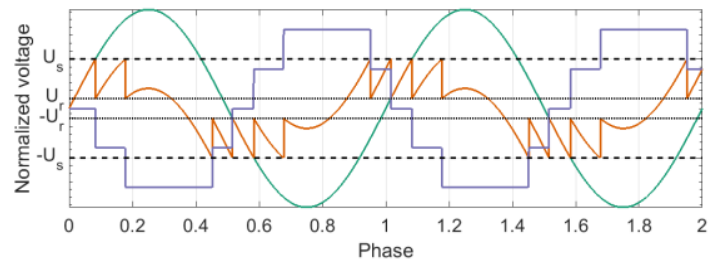

b) Applied voltage at 2.PDIV for the first two periods. $n=4$ per half cycle in the second period when remanent charges are present.

Figure 1: The voltage across a cavity (orange) under the first two periods of an applied AC voltage (green) with voltage from residual charges (purple) opposing the applied voltage. $U_{\mathrm{s}}$ is ignition voltage and $U_{\mathrm{r}}$ the remanent voltage. (a) shows the voltage across the cavity right after PDIV is reached, (b) shows the voltage at twice the PDIV resulting in higher PD repetition rate.

\section{Methodology}

\subsection{Type of test objects}

The test samples used in these experiments were all made up of three equal $1.0 \mathrm{~mm}$ thick sheets of insulation with a cylindrical hole in the middle layer, as illustrated in Figure 2. This resulted in an embedded air-filled disk-shaped cavity, sandwiched between the other two layers. Three different type of test objects were examined:

1. Polycarbonate (Lexan 9030 sheets)

2. Polycarbonate with conducting (provided by a thin layer of aluminum) cavity surfaces

3. New type of generator insulation (Samicatherm - mica and glass fiber reinforced epoxy insulation tape)

The main purpose of performing tests using discs of polycarbonate was to compare the effect of PD characteristics of voids with conductive and insulating surfaces. A thin layer of aluminum was vacuum deposited to the adjacent void surfaces of object type 2. To compare these observations to that of

\begin{tabular}{|c|c|c|c|}
\hline \multicolumn{4}{|c|}{$100 \mathrm{~mm}$} \\
\hline \multicolumn{4}{|l|}{$1 \mathrm{~mm}$} \\
\hline & $1 \mathrm{~mm}$ & Air-filled cavity & $>$ Conducting surface \\
\hline \multicolumn{4}{|l|}{$1 \mathrm{~mm}$} \\
\hline & & $3-20 \mathrm{~mm}$ & \\
\hline
\end{tabular}
generator stator insulation test object 3 was made from laboratory manufactured discs, using the new type of mica/glassfiber reinforced epoxy type. In all cases the diameter of the voids were varied in the range from 3 to $20 \mathrm{~mm}$.

Figure 2: Cross-section of the center of the test objects. The areas marked with "conducting surface" is only for object 2 , whereas the other object have all void surfaces insulating. 


\subsection{Electric field calculation}

The electric field distributions in the cylindrical voids, similar to those presented in Figure 2, were calculated by FEM COMSOL simulations. The main purpose of this was to examine the effect of void diameter on the local electric field magnitude within the void. From a theoretical point of view, the magnitude of the average electric field is expected to strongly affect the PD inception voltage.

\subsection{Electrode arrangement and PD test equipment}

The test samples were clamped between two brass electrodes, as seen in Figure 3. The epoxy-encapsulated high voltage electrode had a diameter of $30 \mathrm{~mm}$. Silicon grease was applied between the electrodes and the sample to prevent any unintended voids caused by sample roughness.

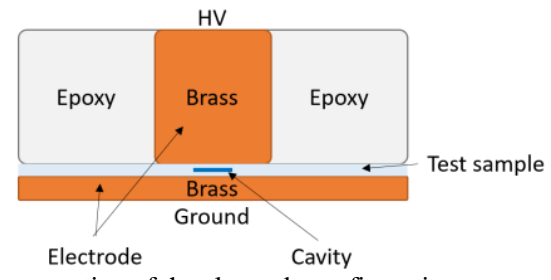

Figure 3: Cross-section of the electrode configuration.

The electrodes together with test object were placed in a PD detection circuit, using the so-called "direct method", shown in Figure 4. The $50 \mathrm{~Hz}$ voltage was applied using a TREK 20/20B High Voltage amplifier. High frequency noise was removed using a low pass filter and a coupling capacitor $\mathrm{C}_{\mathrm{k}}=1200 \mathrm{pC}$ was connected in parallel with the test object. Voltage application and PD detection were controlled by a computer. A measuring impedance was connected in series with the coupling capacitor and a PD detection system, MPD 600 from Omicron, was calibrated with respect to apparent charge and used to analyzing the pulses. Digital PC noise interference was prevented by using a fiber optical signal cables between the master unit MCU 502 and the PC computer. All PDs above the threshold value at $50 \mathrm{pC}$ were included in the analysis.

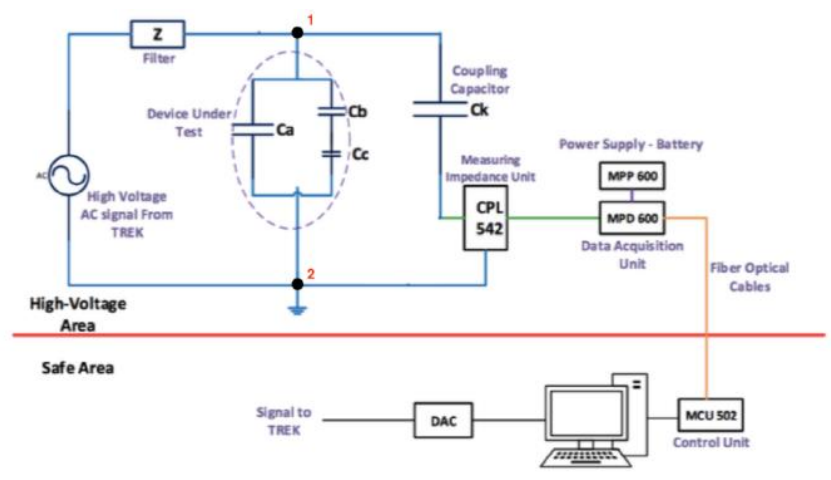

Figure 4: Measuring circuit for the Partial Discharge measurements.

\subsection{PD-Test Procedure}

An initial AC voltage screening revealed that a maximum test voltage of $7 \mathrm{kV}_{\mathrm{RMS}}$ was sufficient to reach PDIV for all examined test objects, and this value was therefore chosen as the level of maximum test voltage. It was decided to energize the test object using a procedure corresponding to IEC 6003427:2006 [7]. This means that all samples were pre-conditioned for $5 \mathrm{~min}$ at the maximum test voltage $\left(7 \mathrm{kV}_{\mathrm{RMS}}\right)$ prior the PD measurement procedure. The PD inception and extinction voltages were then immediately respectively measured during a stepwise increase of voltage from 0 to $7 \mathrm{kV}_{\mathrm{RMS}}$ followed by a similar stepwise decrease, as shown in Figure 5. Each voltage step was $0.5 \mathrm{kV}_{\mathrm{RMS}}$ in magnitude and the duration of each step was set to $30 \mathrm{~s}$. The reported voltage values were taken as the voltage between the steps.

Results from preliminary experiments showed that the aluminum-covered surfaces were severely erode due to the high energy of the PD activity. The pre-conditioning electrification was therefore omitted for these samples as the purpose of the pre-conditioning was to ensure equal and stable initial surface conditions of the insulating surfaces. (Possible gas changes or residual charges from the pre-conditioning were not considered.)

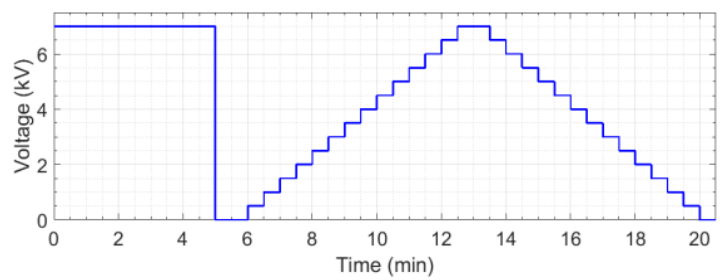

Figure 5: Electrification procedure. Steps of $0.5 \mathrm{kV}_{\mathrm{RMS}}$ for $30 \mathrm{~s}$ from $0 \mathrm{kV}$ to $7 \mathrm{kV}_{\mathrm{RMS}}$ were used. The five-minute pre-condition was not used for the samples with aluminum-coated surfaces in sample 2.

\section{Results from material characterisation and electric field calculations}

\subsection{Relative permittivity of tested insulations}

The capacitances of the different materials were measured by IDAX 206 at $50 \mathrm{~Hz}$ and the relative permittivities of polycarbonate and the stator insulation was determined to 3.4 and 4.2 respectively.

\subsection{Electric field inside voids of different diameters}

The results from FEM calculations of the electric field within the void with insulating surfaces, enclosed in polycarbonate test object 1, is presented in in Figure 6. The field magnitude is here

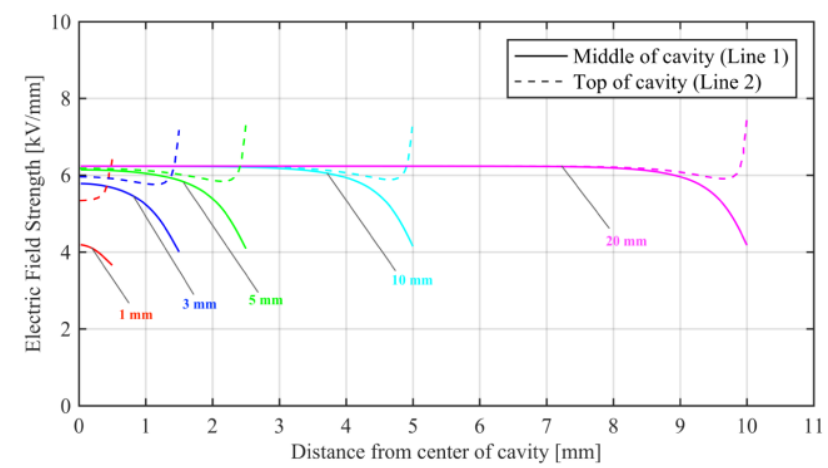

Figure 6: Electric field along Line 1 and Line 2 within voids with insulating surfaces of different diameters, enclosed in polycarbonate (test object 1). 
plotted radially along straight lines from the center of the void, in the middle of the void (line 1) and at the top of the void (line 2). The graphs show that the average electric field in the center of the void is increasing with increasing diameter up to about a diameter of about $10 \mathrm{~mm}$. Similar variations and electric field magnitudes were found in case of void surfaces covered with conductive aluminum. However, with a wider central region of homogeneous field and a somewhat larger field enhancement at the edges.

\section{Results from PD measurements}

\subsection{Comparison of PDIV and PDEV}

Results from measurements of PD inception (PDIV) and extinction voltages (PDEV) for the examined test objects are shown in Figure 7. In all cases nearly no difference was observed between PDIV and PDEV. The theoretical graph based upon Paschen law was adjusted to the calculated average electric field in the voids. The measured values of PDIV agree well with the theoretical/expected values of PDIV. A remanent voltage of about $90 \%$ of the start voltage $U_{\mathrm{s}}$ is needed to provide a PDEV value close to that of PDIV.

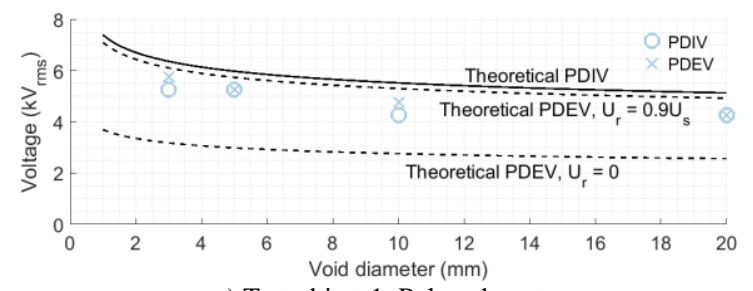

a) Test object 1: Polycarbonate

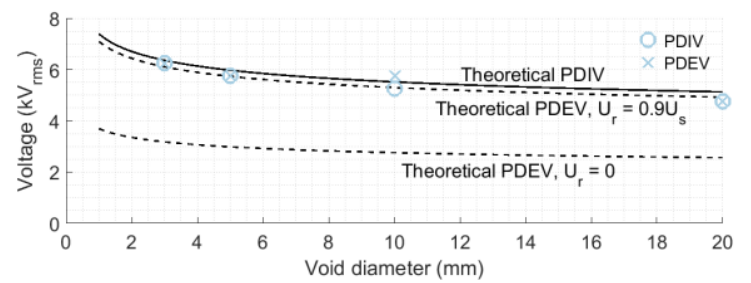

b) Test object 2: Polycarbonate with conducting surfaces

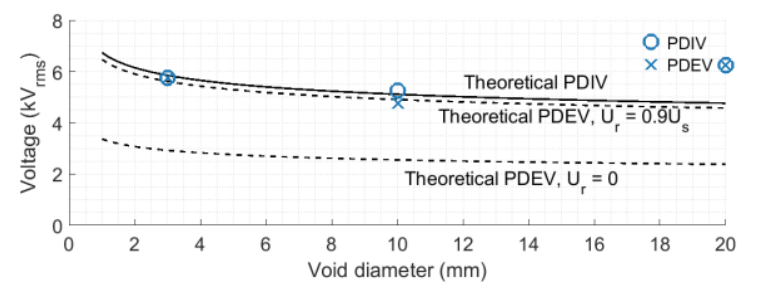

c) Test object 3: Samicatherm - mica and glass fiber reinforced epoxy

Figure 7: PDIV as a function of sample void diameter for the test objects. The error is $\pm 0.25 \mathrm{kV}$. Theoretical values for PDIV are given in the solid line based on Paschen voltage, adjusted by the average electric field in the void, which is diameter dependent. The expected PDEV is given by a dotted line for a remanent voltage of 0 and $90 \%$ of the ignition voltage $U_{\mathrm{s}}$.

\subsection{Maximum apparent charge at PDIV}

The results from measurements of apparent charge values at PDIV are shown in Figure 8 as a function of void diameter. For comparison the theoretical expected values are presented for both the polycarbonate and the stator insulation. In case of voids smaller than $10 \mathrm{~mm}$ in diameter a good agreement between measured and expected charge magnitudes was found. The error of using the approximation in equation (5) compared to equation (4) had less than $5 \%$ deviation. This deviation became about $25 \%$ for the largest voids. As expected, values measured using test objects of type 2 , with conductive void surfaces, were found to fit nearly perfect with the expected theoretical value. In case of the largest void ( $20 \mathrm{~mm}$ in diameter) the measured values were in all cases found lower than the expected apparent charge at PDIV.

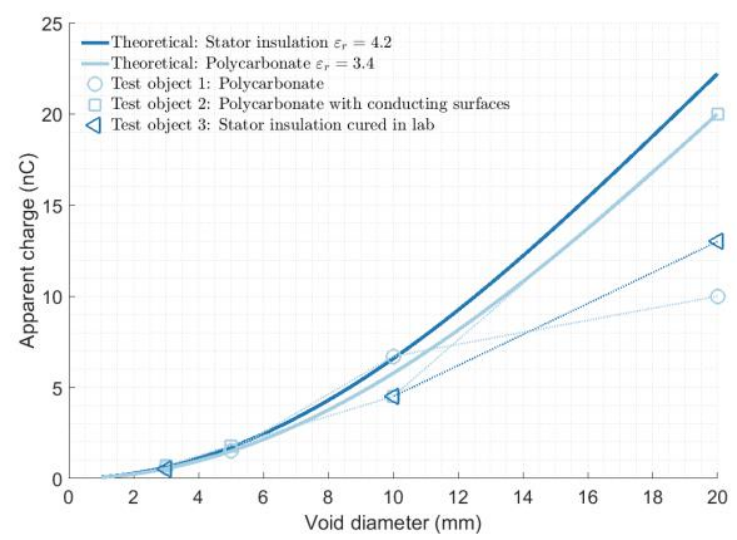

Figure 8: Measured maximum apparent charge at PDIV as function of the void diameter compared to the theoretical values given by equation (4).

\subsection{PD repetition rate and phase resolved patterns}

The results from measurements of the average number of discharges per half period (the PD repetition rate) at PDIV and PDEV is shown in Figure 9 as a function of void diameter. The values are compared to the theoretical value of 2 at PDIV and 1 at PDEV.

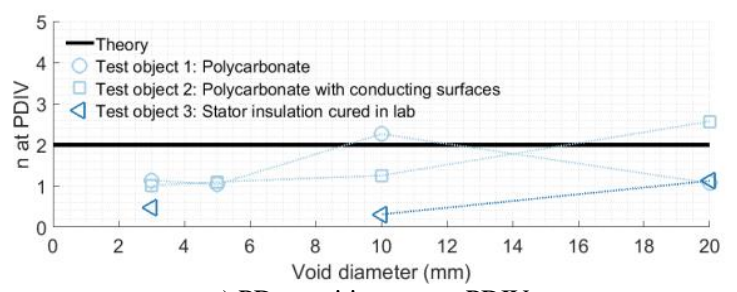

a) PD repetition rate at PDIV

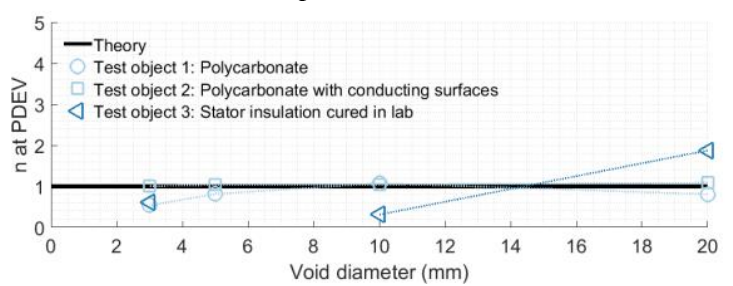

b) PD repetition rate at PDEV

Figure 9: PD number $n$ per half period for the different samples as function of void diameter.

The PD repetition rate as function of voltage for different void diameter in object type 1 are given in Figure 10. Equation (3) was used as theoretical baseline with the remanent voltage varied to fit the data for the different void diameters. 


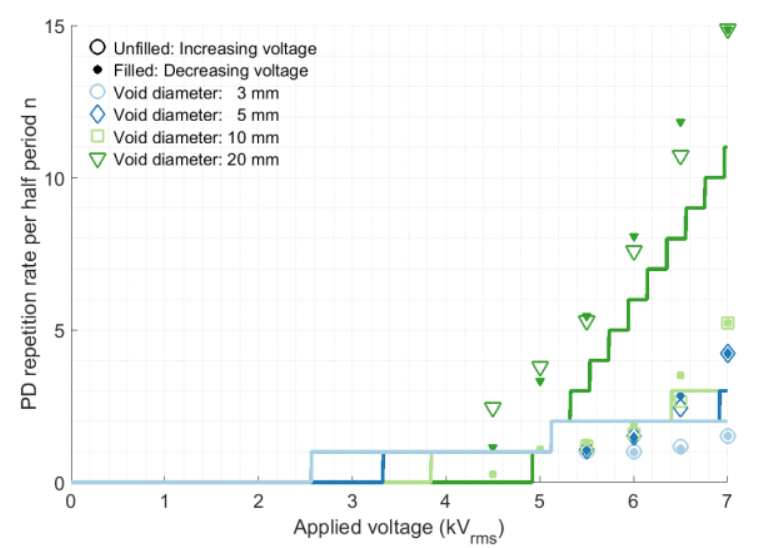

Figure 10: PD repetition rate for different diameters of test object 1 as function of void diameter. The remanent voltages used in the theoretical model, equation (3), (solid lines) are $0,30,50$ and $92 \%$ of the ignition voltage determined by the Paschen curve.

Examples of observed variations in PD magnitudes of polycarbonate test objects, together with the phase locations, are visualized by the PRPD patterns shown in Figure 11. The different patterns due to insulating and conductive void surfaces are clearly demonstrated. In case of aluminum covered conductive void surfaces the apparent charge magnitudes were found to be higher and more constant than in the case of insulating surfaces.
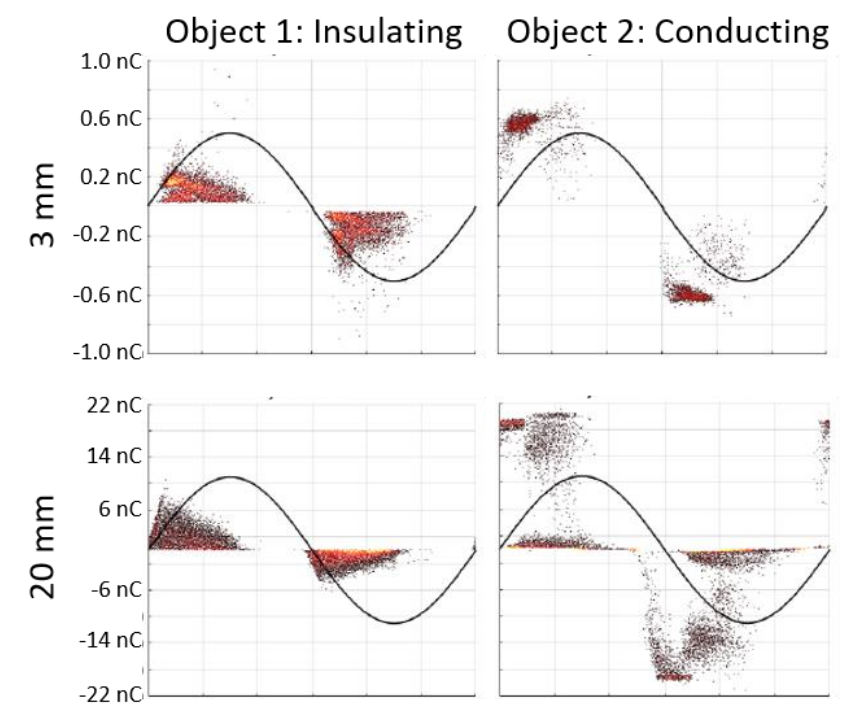

Figure 11: PRPD patterns with recording time of $30 \mathrm{~s}$ at $50 \mathrm{~Hz} 7 \mathrm{kV}_{\mathrm{RMS}}$ (1.2.PDIV). The color represents intensity.

\section{Discussion}

The apparent charge measured in case of test objects with insulating voids up to $10 \mathrm{~mm}$ in diameter were found to be in good agreement with the theoretical expression of equation (5). This indicates that in case of insulating voids smaller than this, the surface area involved in each discharge is not limited by the conductivity of the surface. This statement cannot, however, explain the large spread in apparent charge magnitudes observed in PRPDA pattern of insulating voids. The characteristic difference of the PRPDA pattern between test objects with insulating and conducting void surfaces is that at maximum test voltage the observed charge magnitude of insulating voids is reducing towards increasing test voltage. A phenomenon, which possibly can be explained by high and rapidly increasing values of the remanent voltage.

Observed and estimated numbers of partial discharges per half period according to equation (3) are summarized in Figure 10. It is seen that the effect of increasing the remanent voltage is to increase the number of partial discharges, and subsequently causing reduced magnitude of each discharge. This implies that the remanent voltage may vary between a low and a high value, dependent on the duration of PD activity and the apparent charge magnitude.

This is in contradiction to results presented in the literature indicate that remanent charges decay rather slowly [8]. If this is the case, it is reasonable to assume no decay during a $50 \mathrm{~Hz}$ half period. In case of low surface conductivity, the measured PDIV values becomes highly affected by the remanent charge caused by PD activity during the pre-condition period. A phenomenon, which can explain the small difference between the observed PDIV and PDEV values. However, the relatively small difference between theoretical PDIV and measured PDIV in Figure 7 does not support this. Remanent charges from the precondition period would have significantly lowered the PDIV closer to what is expected by a low remanent voltage for PDEV. The measurements rather suggest that the PDEV is increasing to the PDIV values. This can be explained by a significant residual charge decay. The decay decreases the PD repetition rate at PDIV from 2 to 1 , as can be seen in the simulated example in Figure 12 with a large relaxation rate with the applied voltage close to PDIV. The reduction in repetition rate at PDIV is confirmed by measurements presented in Figure 9a.

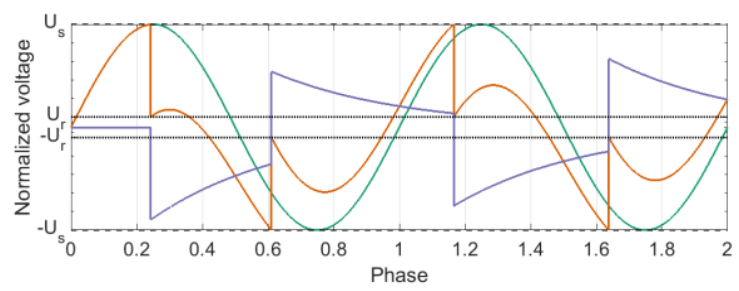

Figure 12: The voltage across a cavity (orange) under the first two periods of an applied AC voltage (green) with voltage from residual charges (purple). $U_{\mathrm{s}}$ is ignition voltage and $U_{\mathrm{r}}$ the remanent voltage. The remanent charges are here assumed to decay and the voltage produced by the charges are decaying, hence an increasing remanent void voltage.

In case of insulating voids with diameters larger than $10 \mathrm{~mm}$ higher PD repetition rate was observed than in case of conductive void surfaces. It is reasonable to assume that this is caused by several PDs occurring in parallel, during which a section of the void surface is discharged only. Thus, it becomes difficult to relate the number of PD measured to the total number of voids in a test object.

\section{Conclusions}

Larger voids resulted in higher number of PD each at lower apparent charge magnitude than expected, indicating several parallel discharges, during which a section of the void surface is discharged only. 
Thus, the abc-model assumption of each PD causing a complete void discharging is only fulfilled if the surfaces are conducting, or the voids are small with nearly homogeneous electric field stress.

In contradiction to the assumptions of the abc-model nearly no difference was observed between the PD inception and extinction voltage, indicating a high and rapidly decaying remanent voltage.

The results indicate that changes of internal conditions of the void during the period of PD measurements, need to be considered in a more realistic model.

\section{Acknowledgement}

This work is founded by the project "Hydrogenerator Stator Winding Insulation Assessment". The project is supported by The Research Council of Norway (Project No. 255099/E20), and industrial partners.

\section{References}

[1] C. Hudon and M. Bélec, "Partial discharge signal interpretation for generator diagnostics," IEEE Transactions on Dielectrics and Electrical Insulation, Article vol. 12, no. 2, pp. 297-319, 2005.

[2] R. Bodega, P. H. F. Morshuis, M. Lazzaroni, and F. J. Wester, "PD Recurrence in Cavities at Different Energizing Methods," IEEE Transactions on Instrumentation and Measurement, Article vol. 53, no. 2, pp. 251-258, 2004.

[3] G. C. Stone, "A perspective on online partial discharge monitoring for assessment of the condition of rotating machine stator winding insulation," IEEE Electrical Insulation Magazine, Review vol. 28, no. 5, pp. 8-13, 2012

[4] E. Kuffel and W. S. Zaengl, High-voltage engineering: fundamentals. Pergamon Press, 1984.

[5] R. Bartnikas and G. L. d'Ombrain, "A Study of Corona Discharge Rate and Energy Loss in Spark Gaps," IEEE Transactions on Power Apparatus and Systems, Article vol. 84, no. 9, pp. 770-779, 1965.

[6] B. Fruth and L. Niemeyer, "The Importance of Statistical Characteristics of Partial Discharge Data," IEEE Transactions on Electrical Insulation, Article vol. 27 , no. 1 , pp. 60-69, 1992.

[7] IEC TS 60034-27:2006, Off-line partial discharge measurements on the stator winding insulation of rotating electrical machines, 2006.

[8] L. A. Dissado, V. Griseri, W. Peasgood, E. S. Cooper, K. Fukunaga, and J. C. Fothergill, "Decay of space charge in a glassy epoxy resin following voltage removal," IEEE Transactions on Dielectrics and Electrical Insulation, vol. 13, no. 4, pp. 903-916, 2006. 